\title{
Omitting Completion Dissection in Melanoma? Help is Available for Surgeons Coping Without Routine Dissection, But More Work is Needed
}

\author{
Jamie Green Rand, MD ${ }^{1}$ and Mark B. Faries, MD, FACS ${ }^{2}$ \\ ${ }^{1}$ Department of Surgery, Cedars Sinai Medical Center, Los Angeles, CA; ${ }^{2}$ Department Surgical Oncology, The Angeles \\ Clinic and Research Institute, Los Angeles, CA
}

The pace of change in therapy for patients with stage 3 melanoma during the last year has been mindboggling. Although the indications clearly showed that we were entering a new therapeutic era, the degree to which "routine" care of stage 3 patients has evolved is remarkable. This change has revolved around two issues: the surgical management of patients with melanoma metastases in sentinel lymph nodes and the utility of adjuvant systemic therapy.

The first issue has seen a large influx of data from two multicenter clinical trials. Both the DeCOG-SLT and the Multicenter Selective Lymphadenectomy Trial II (MSLTII) studies examined completion node dissection, randomizing patients with sentinel node metastases to either the previous standard of completion node dissection or close observation including nodal ultrasound. ${ }^{1,2}$ The trials had remarkably congruent results, with no benefit in terms of distant metastasis-free survival or melanoma-specific survival derived from early dissection. Multiple studies of adjuvant medical therapy informed the second issue, with both BRAF/MEK combination inhibition and anti-PD-1 antibody therapy demonstrating significant benefits in the adjuvant setting for stage 3 melanoma. ${ }^{3-5}$ Although it is wonderful to see this type of progress, we are left with many unanswered questions about what currently constitutes "optimal" treatment.

(C) Society of Surgical Oncology 2018

First Received: 29 June 2018;

Published Online: 12 September 2018

M. B. Faries, MD, FACS

e-mail: mfaries@theangelesclinic.org
In this issue of the Annals of Surgical Oncology, Sinnamon et al. $^{6}$ report data that contribute to the community's ongoing discussions. Using their institutional database, they selected patients with sentinel node metastases and searched for factors associated with non-sentinel node metastases found at the time of completion lymph node dissection. Among their patients $16 \%$ had such nonsentinel node metastases, which were related to both tumor factors (Breslow thickness $\geq 3 \mathrm{~mm}$, absence of tumor-infiltrating lymphocytes) and sentinel nodal factors (metastasis size $\geq 1 \mathrm{~mm}$, combined subcapsular/parenchymal metastasis location, proportion of positive sentinel nodes $\geq 2 / 3$ ). Predicted rates of non-sentinel node metastases varied from $1 \%$ for patients with a score of zero to $60.6 \%$ for the highest-risk patients. The scheme performed well, with close correspondence to observed rates of non-sentinel node metastases, albeit in the same population from which the score was derived. The authors concluded that "high-risk patients may warrant adjuvant therapy or more intensive surveillance of the nodal basin to reduce risk of loss of regional control."

This information certainly is useful as we grapple with the decreasing availability of complete pathologic staging for stage 3 patients who opt for observation of their regional basin, but we should consider any conclusions with some caution. The result here is similar in many ways to that of prior efforts at other institutions, many of which showed the extent of primary tumor or nodal involvement that correlated with non-sentinel node disease.$^{7-9}$ However, there has been limited consistency in the specific measures assessing the extent of disease across institutions.

Nodal disease burden has been quantified in several ways including maximum diameter of the largest metastatic focus, percentage of nodal area involvement, depth of 
nodal penetration, and metastasis location. Comparisons of these methods have been limited, and their conclusions have not been uniform. The most straightforward method and the one most commonly used is maximum metastasis diameter, but even with this method, studies differ regarding the most relevant cutoff for high or low risk. The 1-mm cutoff used in this study has been the most common and currently is an eligibility criterion in multiple adjuvant therapy trials. Based on analyses of the current (8th edition) and prior (7th edition) American Joint Committee on Cancer (AJCC) databases, there does not appear to be a minimum tumor volume below which nodal metastases can be discounted. ${ }^{10}$ Independent validation of this score will be important. ${ }^{11,12}$ Also, as the authors suggested, we should be quite careful about how we interpret the use of nodal ratio in the scoring system. Although this variable seems strongly related to non-sentinel node metastases, it should in no way be used to suggest that removing a larger number of sentinel nodes is desirable. Any melanoma will have a certain number of nodes receiving direct drainage from the primary site, and that number is determined by the patient's anatomy, not the surgeon's technique.

On a more conceptual basis, it also is important to note that a pathologically negative completion dissection specimen cannot be interpreted as the absence of non-sentinel node metastases. It appears that we miss a substantial fraction of non-sentinel node metastases during routine pathologic assessment of completion lymph node dissection specimens, which generally does not include stepsectioning or immunohistochemistry. In MSLT-II, the rate of non-sentinel node metastases detected on pathology in the completion node dissection arm was $11.5 \%$, whereas the rate of in-basin recurrence in the observation arm was $22.9 \%$ at 3 years. $^{2}$ Therefore, it is possible that half of the patients with non-sentinel node metastases go undetected when standard pathologic processing is used, and we must be cautious in making strong recommendations with the data available to date.

The other aspect of melanoma care that has been revolutionized in the last year is adjuvant therapy with the approval of multiple effective targeted and immune agents for patients with completely resected stage 3 disease. However, these agents are costly and carry the risk of potentially serious side effects. One clear benefit of completion node dissection is the provision of prognostic information shown to be both powerful and independent of other variables including tumor thickness and ulceration. This information may help patients who are undecided about whether to undergo medical therapies in making a decision. The adjuvant therapy trials leading to these recent approvals required completion node dissection for eligibility and required either stage $3 \mathrm{~B}$ or greater disease or sentinel node metastasis with a maximum diameter of at least $1 \mathrm{~mm} .^{3-5}$ This means that a large proportion of stage 3 patients in the modern era without completion node dissection would not have met the eligibility requirements for the trials.

Fully informing patients about their risk of recurrence and death when they are considering adjuvant therapy is challenging without complete nodal staging. Can this new scoring system replace the prognostic information of completion dissection? Although the authors report a correlation of the score with melanoma-specific survival, we currently do not seem we have sufficient information to know this. New clinical trials, or at least reevaluation of existing adjuvant trial data, could help clarify the answer to that question. In addition, it will be interesting to see how these scores fare in predicting nodal recurrence in patients opting for surveillance of the nodal basin.

The adjuvant systemic therapy trials that led to currently approved drugs were stratified based on nodal stage, which requires complete dissection to be accurate, and the primary end point of those trials was recurrence-free survival. $^{3-5}$ If most patients no longer undergo complete dissection, there may be a risk for imbalance of early recurrences based on retained nodal disease that would have been removed in the earlier trials. The design of any new adjuvant trials will have to take this potential complication into account, and distinguishing regional nodal recurrences from distant metastases will be important when the trials are analyzed.

In 2018, who, if anyone, should be encouraged to undergo a completion lymph node dissection? High-level evidence provides reassurance that close nodal observation is safe for most patients, but confidence in this safety requires that observation actually is close. Patients who cannot follow the type of schedule used in the prospective studies are probably not good candidates for omitting surgery. What about patients at high risk or with high scores? If we had a system to predict non-sentinel node metastasis with $100 \%$ certainty, immediate dissection would seem to be the best standard choice because it would have a dramatic impact on regional recurrence and diseasefree survival overall. Because elective dissection for microscopic disease seems to carry a lower risk of morbidity than delayed therapeutic dissection for clinically positive disease, early intervention also would be likely to improve the quality of life for patients with such disease. ${ }^{13}$ However, it appears that we still are not at that level of accuracy, and to date, it does not appear that high-risk subgroups (however that is defined) are more likely to derive a melanoma-specific or overall survival benefit from immediate dissection. Additionally, the question of benefit for high-risk groups may become even cloudier as the use of adjuvant therapy for stage 3 melanoma increases. As a 
community, we must work hard to explore the new areas of ignorance that have been uncovered by the whirlwind of progress in melanoma this year.

FUNDING Funding was provided by National Cancer Institute (5R01CA189163).

\section{REFERENCES}

1. Leiter U, Stadler R, Mauch C, et al. Complete lymph node dissection versus no dissection in patients with sentinel lymph node biopsy positive melanoma (DeCOG-SLT): a multicentre, randomised, phase 3 trial. Lancet Oncol. 2016;17:757-67.

2. Faries MB, Thompson JF, Cochran AJ, et al. Completion dissection or observation for sentinel-node metastasis in melanoma. $N$ Engl J Med. 2017;376:2211-22.

3. Weber J, Mandala M, Del Vecchio M, et al. Adjuvant nivolumab versus ipilimumab in resected stage III or IV melanoma. $N$ Engl $J$ Med. 2017;377:1824-35.

4. Long GV, Hauschild A, Santinami M, et al. Adjuvant dabrafenib plus trametinib in stage III BRAF-mutated melanoma. $N$ Engl $J$ Med. 2017;377:1813-23.

5. Eggermont AMM, Blank CU, Mandala M, et al. Adjuvant pembrolizumab versus placebo in resected stage III melanoma. $N$ Engl J Med. 2018;378:1789-801.

6. Sinnamon AJ, Song Y, Sharon CE, et al. Prediction of residual nodal disease at completion dissection following positive sentinel lymph node biopsy for melanoma. Ann Surg Oncol. 2018. http s://doi.org/10.1245/s10434-018-6647-7.
7. Cochran AJ, Wen DR, Huang RR, et al. Prediction of metastatic melanoma in nonsentinel nodes and clinical outcome based on the primary melanoma and the sentinel node. Mod Pathol. 2004;17:747-55.

8. Murali R, Desilva C, Thompson JF, et al. Non-sentinel node risk score (N-SNORE): a scoring system for accurately stratifying risk of non-sentinel node positivity in patients with cutaneous melanoma with positive sentinel lymph nodes. $J$ Clin Oncol. 2010;28:4441-9.

9. Frankel TL, Griffith KA, Lowe L, et al. Do micromorphometric features of metastatic deposits within sentinel nodes predict nonsentinel lymph node involvement in melanoma? Ann Surg Oncol. 2008;15:2403-11.

10. Gershenwald JE, Scolyer RA, Hess HR, et al. Melanoma staging: evidence-based changes in the American Joint Commission on Cancer Eighth Edition Cancer Staging Manual. CA Cancer J Clin. 2017;67:472-92.

11. McMasters KM, Wong SL, Edwards MJ, et al. Frequency of nonsentinel lymph node metastasis in melanoma. Ann Surg Oncol. 2002;9:137-41.

12. Wevers KP, Murali R, Bastiaannet E, et al. Assessment of a new scoring system for predicting non-sentinel node positivity in sentinel node-positive melanoma patients. Eur J Surg Oncol. 2013;39:179-84.

13. Faries MB, Thompson JF, Cochran A, et al. The impact on morbidity and length of stay of early versus delayed complete lymphadenectomy in melanoma: results of the Multicenter Selective Lymphadenectomy Trial (I). Ann Surg Oncol. 2010;17:3324-9. 